\title{
Compostos Inorgânicos Como Fármacos
}

\section{Mônica Aparecida Rodrigues, Reinaldo Ruggiero e Wendell GuerRA*}

\begin{abstract}
Este artigo aborda de forma sucinta a aplicação de compostos inorgânicos como fármacos, dando um enfoque especial para os compostos de platina, bismuto e prata.
\end{abstract}

Palavras-chave: Química inorgânica medicinal; complexos metálicos; agentes antitumorais

Inorganic compounds as drugs: This article discusses briefly the application of inorganic compounds as drugs, with a special focus on the compounds based on platinum, bismuth and silver.

Keywords: Medicinal inorganic chemistry; metal complexes; anticancer agents

\section{INTRODUÇÃO}

As inúmeras actividades biológicas exercidas por iões metálicos têm recentemente estimulado o desenvolvimento de fármacos à base de metais (metal-based drugs). Embora o uso de compostos inorgânicos para o tratamento de enfermidades seja muito antigo, até à descoberta da actividade antitumoral do complexo denominado cisplatina, $\left[\mathrm{Pt}\left(\mathrm{NH}_{3}\right)_{2} \mathrm{Cl}_{2}\right]$, por Rosenberg e colaboradores em 1965, não havia grande interesse em sintetizar ou entender as bases moleculares responsáveis pelo mecanismo de acção destes compostos. De facto, a descoberta de Rosenberg impulsionou a pesquisa e o desenvolvimento de fármacos à base de metais [1, 2]. A introdução da cisplatina nas práticas médicas em 1978 provocou uma corrida científica no sentido de obter novos compostos com melhor actividade antitumoral, menor toxicidade e maior espectro de acção, sobretudo no que diz respeito às células resistentes à cisplatina. Assim, desde 1965 até hoje, foram lançados no mercado seis novos complexos de platina dos quais podemos destacar a carboplatina que possui menor efeito nefrotóxico do que a cisplatina, podendo ser administrada em doses maiores. Além da cisplatina e da carboplatina, os outros complexos de platina disponíveis para tratar diversos tipos de cancro são a oxaloplatina, nedaplatina,

Instituto de Química, Universidade Federal de Ubelândia, Campus Santa Mônica, Bloco 1D

* e-mail: wg@iqufu.ufu.br
sunpla/SK-2053R e lobaplatina [2,3]. Em relação a outros fármacos à base de metais, há actualmente vários compostos inorgânicos que são utilizados na medicina moderna, dentre os quais podemos destacar os complexos de gadolínio(III), que são utilizados como agentes de contraste em ressonância magnética, os de tecnécio-99, que são usados na obtenção de imagens cardiovasculares, e os de ouro, ferro e bismuto, que são utilizados no tratamento da artrite reumatóide, hipertensão e úlceras pépticas, respectivamente [2-6]. $\mathrm{Na}$ Figura 1 e na Tabela I listam-se vários compostos inorgânicos actualmente disponíveis como fármacos.

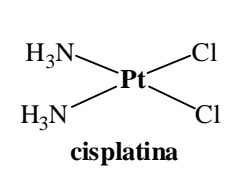<smiles>N[P+]1(N)OCC(=O)O1</smiles>

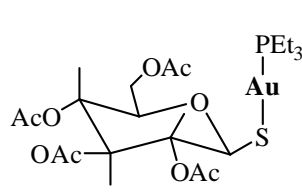

Auranofina<smiles>NP1(N)(N)OC(=O)C2(CCC2)C(=O)O1</smiles><smiles>CO[P+](N)(N)OC(=O)C1CCCCC1</smiles>

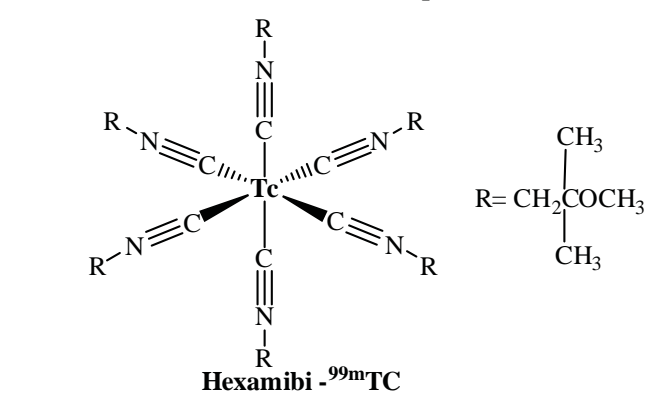<smiles>CC(C)N(c1ncccn1)S(=O)(=O)c1ccc(N)cc1</smiles>

Figura 1 Estrutura de alguns complexos metálicos utilizados na clínica médica 
Tabela 1 Compostos inorgânicos utilizados no tratamento e diagnóstico de doenças

\begin{tabular}{ccc}
\hline Composto & Uso clínico/função* & Comentário \\
\hline $\mathrm{Li}_{2} \mathrm{CO}_{3}$ & $\begin{array}{c}\text { Tratamento de perturbação } \\
\text { bipolar } \\
\mathrm{CaCO}_{3} \mathrm{e}\end{array}$ & \\
$\mathrm{Mg}(\mathrm{OH})_{2}$ & Antiácidos & \\
$\mathrm{La}_{2}{ }^{\text {III }}\left(\mathrm{CO}_{3}\right)_{3}$ & Falência renal crónica & Age reduzindo a absorção \\
$\mathrm{Fosrenol}^{\mathrm{MM}}$ & de fosfato \\
$\mathrm{BaSO}_{4}$ & Agente de contraste & \\
\hline
\end{tabular}

Além dos complexos citados, que são amplamente utilizados como medicamentos, há uma variedade de compostos inorgânicos que estão a ser investigados em relação ao seu possível uso em clínica médica. Dentre os mais promissores como agentes antitumorais estão os complexos de ruténio, gálio, ouro, titânio e platina [7-11]. Outros complexos promissores são os de vanádio para tratamento da diabetes [10] e os de paládio(II) como agentes anti-infecciosos [12, 13].

A coordenação de metais a fármacos e moléculas que não são utilizadas como medicamento representa um potencial considerável para aumentar o arsenal de medicamentos disponíveis para o tratamento de enfermidades [14, 15]. Entretanto, o desenvolvimento de fármacos, sejam eles orgânicos ou inorgânicos, não é uma tarefa fácil. No caso específico de compostos inorgânicos, a bioacumulação do ião metálico pode causar efeitos colaterais graves e, portanto, devem ser investigados aspectos farmacológicos e fisiológicos in vivo utilizando modelos animais que comprovem a eficácia e segurança do composto antes da libertação do fármaco para testes clínicos em humanos [6]. É interessante comentar que recentemente o medicamento Fosrenol ${ }^{\mathrm{TM}}\left(\mathrm{Ln}_{2}\left(\mathrm{CO}_{3}\right)_{3}\right)$ foi autorizado pela FDA (Food and Drug Administration-USA) para uso clínico no tratamento de pacientes que sofrem de falência renal crónica. O fármaco, que age reduzindo a absorção de fosfato, é considerado uma droga simples, eficaz e segura [7].

Considerando a relevância do tema, este artigo relata alguns dos avanços recentes no desenvolvimento de complexos de platina, bismuto e prata como agentes terapêuticos promissores.

\section{Desenvolvimento de complexos de PLATINA COMO AGENTES ANTITUMORAIS}

A cisplatina, um complexo com geometria quadrangular plana, foi preparada pela primeira vez em 1844 por Michele Peyrone, mas somente em 1965 é que a sua propriedade antitumoral foi descoberta. Como referido anteriormente, a cisplatina apresenta alguns efeitos colaterais severos como nefrotoxicidade, pelo que a preparação e desenvolvimento de novos complexos de platina que sejam mais eficientes e mais seguros são um estímulo para investigadores da área. De facto, milhares de complexos têm sido sintetizados e testados, mas somente seis são actualmente utilizados em clínica médica [4].

Complexos antitumorais clássicos de platina possuem ligandos lábeis, sendo haletos e carboxilatos os mais comuns, e ligandos estáveis, como por exemplo, aminas, que acompanham o complexo até ao interior da célula onde o mesmo interage com o ADN. Neste sentido, a utilização de diferentes ligandos pode modificar a actividade biológica dos complexos [16]. Além de novos tipos de ligandos, outra estratégia utilizada é a modificação da carga do metal. Os complexos de platina (IV) estão bem estudados, pois são em geral mais solúveis em água quando comparados com os análogos de platina (II), além de possuirem outras características que despertam o interesse no seu estudo [16]. Além disso, os complexos de $\mathrm{Pt}(\mathrm{IV})$ possuem um número de coordenação maior apresentando a possibilidade de serem mais lipofílicos através da inserção de ligandos adicionais que conferem esta característica. Além dos complexos de Pt(II) e $\mathrm{Pt}(\mathrm{IV})$ com geometria cis, os complexos do tipo trans também são alvo de investigação. Durante décadas, estes complexos foram marginalizados devido ao facto de o análogo trans da cisplatina ser inactivo in vivo e significativamente menos citotóxico in vitro quando comparado com a cisplatina. Porém, vários complexos com geometria trans foram obtidos nos últimos anos, os quais demonstraram resultados animadores em relação à actividade citotóxica, pois demonstraram ser activos em linhagens celulares resistentes à cisplatina. Contudo, por enquanto, nenhum destes compostos entrou em fase de testes clínicos [17]. Alguns autores têm especulado que a síntese racional de complexos do tipo trans pode ser uma solução para o problema da resistência intrínseca ou adquirida à cisplatina e, portanto, merecem continuar a ser investigados, até mesmo por que não se sabe ao certo o mecanismo de acção destes compostos [17].

Actualmente, há vários complexos de platina em fase de testes clínicos e outros que embora ainda não estejam em fase de testes clínicos são muito promissores. Um complexo de platina(II) análogo à cisplatina, que é alvo de particular atenção, é o complexo denominado ZDO473 ou AMD473 (complexo I, Figura 2), que apresenta na sua estrutura o ligando 2-metilpiridina e que se encontra na fase III de testes clínicos. Este complexo, em combinação com adriamicina, está a ser avaliado no tratamento de cancro do ovário onde outros fármacos antitumorais semelhantes à cisplatina e paclitaxel não tiveram efeito. Quanto à toxicidade, durante a fase II de testes clínicos não foram observados efeitos tóxicos como nefrotoxicidade, neurotoxicidade periférica ou ototoxicidade. Outros efeitos colaterais como náuseas, vómitos e gosto metálico na boca foram considerados brandos [18-22].

A ausência de efeitos colaterais graves é importante, pois permite que o fármaco possa ser administrado em doses maiores. 
Vários complexos de platina(IV) mostraram ser promissores durante testes realizados in vitro e in vivo, mas apenas o complexo II, Figura 2, conhecido como JM-216 (satraplatina) se encontra na fase III de testes clínicos. A satraplatina pode vir a ser o primeiro composto de platina a ser administrado por via oral e, quando comparado com a cisplatina, apresenta a vantagem de ser menos tóxica [6, 9]. Curiosamente este composto teve os testes interrompidos durante a fase I de testes clínicos por apresentar diferentes modos de mecanismo de acção e uma baixa absorção celular [4]. Outro complexo de platina(IV) bastante promissor é o complexo III, Figura 2 , que é activo em linhagens celulares resistentes à cisplatina e que pode futuramente ser utilizado em casos onde há uma resistência adquirida ou intrínseca à cisplatina [23].

A utilização de complexos metálicos como fármacos é limitada principalmente pela toxicidade, pela degradação do fármaco in vivo e pela pouca solubilidade em água. Neste aspecto, a utilização de sistemas capazes de transportar o fármaco até um alvo específico é uma estratégia que vem contribuindo para resolver estes problemas [24].

As ciclodextrinas (CDs) são uma classe de compostos macrocíclicos livres de toxicidade e de baixo custo, que formam complexos de inclusão com vários fármacos. Como resultado, obtêm-se complexos que são em geral mais solúveis em água, mais estáveis nos meios biólogicos e menos tóxicos [24]. Um exemplo interessante, envolvendo a formação de um complexo de inclusão com ciclodextrinas, é a encapsulação do complexo trans-dicloro(dipiridina)platina(II).

O complexo resultante, Figura 2, complexo IV, tornou-se mais solúvel em água e sua actividade antitumoral melhorou significativamente. Os resultados obtidos mostraram que o complexo de inclusão do tipo 1:1 é 4,6 e 6,1 vezes mais activo in vitro do que a cisplatina nas linhagens celulares CT26 e B16F10, respectivamente [17]. Krause-Heur et al [25] obtiveram complexos de inclusão do tipo 1:1 e 1:2 com $\beta$-ciclodextrina substituída e demonstraram que ocorre um decréscimo significativo na degradação dos complexos de platina pela L-glutationa reduzida. Além do aumento na estabilidade, os complexos formados permaneceram activos em linhagens celulares (LoVo) de cancro colo-rectal. Estes resultados reforçam a utilização de ciclodextrinas como sistemas transportadores de fármacos nas circunstâncias em que o composto apresente pouca solubilidade em água ou quando é instável nos meios biológicos. Têm sido publicados inúmeros trabalhos na literatura demonstrando as vantagens obtidas nos processos de inclusão [26-28].

Uma outra classe de compostos bastante promissora, no sentido de se obterem novos complexos de platina activos em células resistentes à cisplatina, é aquela em que os complexos contêm duas ou mais unidades de platina ligadas por um ligando diaminado de cadeia carbónica de comprimento variável (grupo espaçador), sendo o grupo lábil composto por cloretos, sulfóxidos ou malonatos [29-31]. Uma característica interessante destes complexos multinucleares é a possibilidade de obtenção de compostos com diferentes grupos a ligarem os centros metálicos, grupos espaçado- res, que vão alterar as propriedades físico-químicas do composto, influenciando o seu modo de acção.

Dentre os compostos obtidos até ao momento, um complexo trimetálico de platina conhecido como BBR3464, complexo V, Figura 2, é considerado bastante promissor, pois testes feitos in vitro demonstraram resultados interessantes, uma vez que o complexo exibiu maior actividade que a cisplatina em linhagens resistentes [32]. Este composto encontra-se na fase II de testes clínicos para tratamento de cancro do estômago e do ovário [33]. A interacção destes compostos contendo dois ou mais centros metálicos com o ADN é apontada como o maior factor para a citotoxicidade, sendo sugerido que ocorre a formação de diferentes aductos com o ADN, podendo ser esta a causa da elevada sensibilidade apresentada por eles em células resistentes à cisplatina [34]. Entretanto, a acumulação de fármaco pode ser um factor determinante para a sua citotoxicidade, e uma especulação é que a concentração intracelular destes compostos seja superior à de cisplatina em células resistentes, ou que a sua taxa de entrada na célula seja maior do que a de cisplatina.<smiles></smiles><smiles>CO[C@@](N)(NC1CCCCC1)[Ge](Cl)(Cl)Cl</smiles>

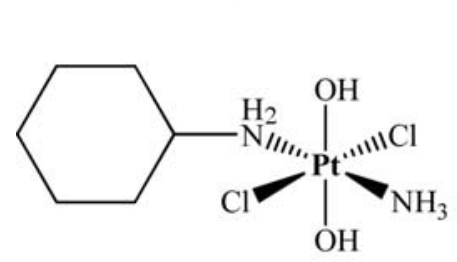

III

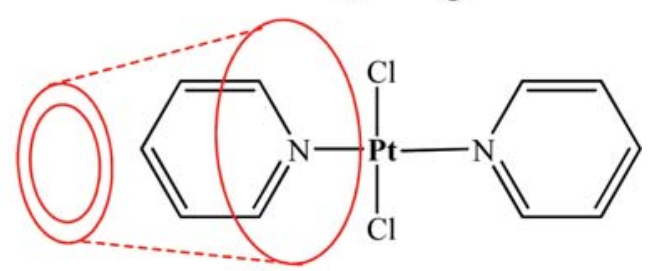

IV<smiles>NCCN[P+](N)(N)NCCN[PH](N)(N)Cl</smiles>

Figura 2 Estrutura de alguns complexos de platina promissores 
USO E DESENVOLVIMENTO DE COMPLEXOS DE BISMUTO COMO AGENTES TERAPÊUTICOS

As úlceras pépticas induzidas pela bactéria Gram-negativa Helicobacter pylori estão entre as infecções mais comuns em seres humanos, afectando aproximadamente $80 \%$ da população em países subdesenvolvidos. Além disso, acredita-se que existe uma relação entre a infecção causada pela $H$. pylori e o cancro gástrico [35]. O tratamento de úlceras pépticas causadas pela $H$. pylori consiste na erradicação da bactéria. Neste aspecto, diversas formulações farmacêuticas contendo complexos de bismuto (De-nol $\circledast$, Pepto-Bismol $\circledast$, Pylorid $囚$ ) são não só utilizadas no tratamento de úlceras pépticas, mas também nos casos de diarreia e dispepsia [36]. Além disso, compostos de bismuto são também utilizados noutras situações como no tratamento da sífilis e cancro [36, 37].

No que respeita ao desenvolvimento de fármacos à base de bismuto, refira-se que complexos de bismuto(III) contendo derivados de tiosemicarbazonas apresentam boa actividade antibacteriana contra as bactérias Gram-positivas Staphylococcus aureus e Bacillus subtilis [38]. A respeito da coordenação de metais em moléculas biologicamente activas, foram obtidos complexos de $\mathrm{Bi}(\mathrm{III})$ contendo antibióticos da família das fluorquinolonas (ciprofloxacina e norfloxacina). O complexo com ciprofloxacina tem potencial uso no tratamento de $H$. pylori [39]. Já o complexo de $\mathrm{Bi}(\mathrm{III})$ contendo a norfloxacina, é mais solúvel em água e mais activo quando comparado quer com a norfloxacina livre quer com a mistura física norfloxacina/citrato de bismuto.

Os resultados deste trabalho demonstram que este complexo é uma alternativa à norfloxacina, principalmente no tratamento de infecções provocadas por Bacillus pumilis (NTCC 8241) e Escherichia coli (ATCC 25922) [40]. É interessante comentar que, recentemente, um medicamento contendo citrato de bismuto coloidal, metronidazol e tetraciclina foi aprovado pela FDA para o tratamento e erradicação da H. pylori [37].
Refira-se que, em relação à actividade antitumoral de compostos de bismuto, estes foram estudados inclusive in vivo contra cancro do sangue (leucemia) em ratos e exibem óptima actividade antitumoral que pode atingir $100 \%$ de cura. Estudos in vitro realizados com diversos complexos de bismuto demonstraram que estes compostos possuem actividade superior à exibida pela cisplatina quando utilizados no tratamento de leucemia e cancro do ovário [41]. Recentemente, foi sugerida a administração de bismuto em associação com a cisplatina, uma vez que alguns compostos de bismuto diminuem a nefrotoxicidade causada pela cisplatina [41]. Iuchi et al [42] sintetizaram um complexo organometálico de $\mathrm{Bi}(\mathrm{III})$ que apresenta forte actividade antibacteriana e antitumoral em linhagens celulares de leucemia e cancro humano. Um complexo de bismuto interessante e muito promissor é o que contém o ligando TPC, Figura 3. Além de ser solúvel em água, ele apresenta excelente actividade citotóxica contra a linhagem de cancro de pele B16-BL6. A inibição de $82 \%$ no crescimento celular deu-se em apenas 48 horas, para uma concentração de $0,25 \mu \mathrm{M}$. O IC (concentração inibitória para $50 \%$ de células) do complexo para a linhagem B16-BL6 é de apenas 41 nM, que é cerca de 100 vezes maior que o da cisplatina [43].

Investigadores do National Cancer Institute dos EUA avaliaram uma série de compostos inorgânicos com potencial actividade antitumoral. Nestes estudos, as relações entre estrutura e actividade são avaliadas pela capacidade do fármaco de inibir o crescimento de células tumorais. Com estes esforços, os investigadores têm sugerido possíveis mecanismos de acção para diversos compostos considerados promissores. No caso dos complexos de bismuto contendo ditiocarbamatos e tropolonas foi sugerido que a actividade antitumoral destes compostos pode estar relacionada com a inibição de enzimas [44]. Apesar de alguns avanços recentes, ainda persistem dúvidas sobre o mecanismo de acção destes compostos e novos estudos devem ser realizados. Em relação ao potencial uso de complexos de bismuto em clínica mé- dica, os ligandos contendo oxigénio e enxofre como átomos doadores são bastante promissores e merecem ser objecto de investigação [38]. Além de complexos de $\mathrm{Bi}(\mathrm{III})$, compostos contendo isótopos de ${ }^{212} \mathrm{Bi}$ e ${ }^{213} \mathrm{Bi}$ têm sido utilizados em rádioterapia para o tratamento de cancro [45].

Relativamente à segurança no emprego destes compostos, os complexos de bismuto são fármacos que possuem baixa toxicidade, tendo como efeitos colaterais mais comuns a disfunção neurológica e a nefrotoxicidade em consequência da sobredosagem. A nefrotoxicidade é diagnosticada pela detecção de bismuto no plasma, soro ou sangue. Os efeitos tóxicos provocados pelo bismuto são reversíveis ao longo de algumas semanas ou meses, quando a ingestão de bismuto é interrompida. Em ensaios com animais, a sobredosagem de bismuto causou uma diminuição nos níveis séricos de testosterona sugerindo que estes compostos podem causar disfunção reprodutiva [38].

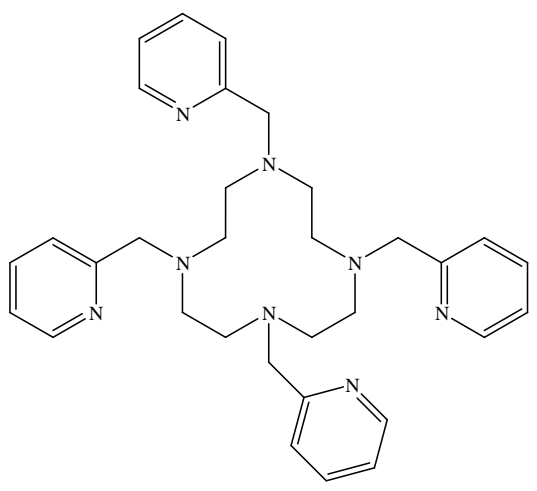

Figura 3 Estrutura do ligando TPC

\section{O USO DE COMPLEXOS DE PRATA COMO AGENTES ANTIMICROBIANOS}

Um bom exemplo de metal coordenado a fármaco é a sulfadiazina de prata, Figura 1, que é muito utilizada em pacientes queimados para evitar e tratar infecções. Este fármaco de uso tópico combina, num só composto, as propriedades antibacterianas do ião prata e da sulfadiazina que é um antibiótico de origem sintética. Como resultado, este composto possui amplo espectro de actividade contra bactérias Gram-positivas e Gram-negativas, sendo muito eficiente contra Pseudomonas aeruginosa que é um dos principais organismos responsáveis por infec- 
ções decorrentes de queimaduras. É interessante notar que sais de prata, como por exemplo o $\mathrm{AgNO}_{3}$, foram utilizados durante séculos como agentes antimicrobianos e que o uso destes compostos diminuiu muito quando os antibióticos foram introduzidos nas práticas médicas. Porém, eles mantiveram-se como um dos agentes mais populares para tratar infecções, especialmente em pacientes queimados. Em virtude do aumento de casos envolvendo resistência aos antibióticos, os compostos de prata, em especial o da sulfadiazina, vêm ganhando espaço [46].

No que se refere ao desenvolvimento de novos compostos, um complexo de prata(I) contendo o sulfametoxazol, que também é um antibiótico da mesma família da sulfadiazina (sulfonamidas) foi avaliado em relação a Staphylococcus aureus, Escherichia Coli e Pseudomonas aeruginosa. Os resultados mostraram que o complexo $\left(\mathrm{IC}_{50}=64 \mu \mathrm{g} \mathrm{mL}^{-1}\right)$ é quatro vezes mais activo nas linhagens $S$. aureus e E. Coli quando comparado com o fármaco livre. Na linhagem $P$. aeruginosa o composto metálico é trinta e duas vezes mais activo que o antibiótico, exibindo um valor de $\mathrm{IC}_{50}$ igual a 16 $\mu \mathrm{g} \mathrm{mL} \mathrm{m}^{-1}$ [47]. Um complexo de prata, também contendo um antibiótico, a norfloxacina, foi obtido e verificou-se que o mesmo pode ser utilizado para prevenir infecções em humanos durante o tratamento de queimaduras. A sua actividade antibacteriana é superior à do fármaco sulfadiazina de prata [48]. Raí et al [49] sintetizaram um complexo de prata (I) do tipo $\left[\mathrm{Ag}_{4}(\mathrm{dppm})_{2}(\mathrm{~L})_{2}\left(\mathrm{NO}_{3}\right)_{2}\right]$ sendo $\mathrm{dppm}=$ bis(difenilfosfina)metano e $\mathrm{HL}_{2}=[(\mathrm{E})$ 3-(4-(9H-carbazol-9-il)fenil)ácido acrílico] e a sua actividade antibacteriana foi avaliada em relação a duas bactérias Gram-positivas (Bacillus subtilis ATCC 6633 e Staphylococcus aureus ATCC 6538) e duas bactérias Gram-negativas (Pseudomonas aeruginosa ATCC 13525 e Escherichia coli ATCC 35218).

O complexo $\left[\mathrm{Ag}_{4}(\mathrm{dppm})_{2}(\mathrm{~L})_{2}\left(\mathrm{NO}_{3}\right)_{2}\right]$ apresenta forte actividade antibacteriana contra a linhagem $B$. subtilis ATCC 6633 ( MIC = 0,78 $\mu \mathrm{g} / \mathrm{mL}$ ) sendo superior à actividade do antibiótico penicilina (MIC = 1,562 $\mu \mathrm{g} / \mathrm{mL}$ ).
A respeito do uso da prata como agente antibacteriano e antifúngico, estudos promissores indicam que nanopartículas de prata (NPs) podem ser utilizadas contra bactérias Gram-positivas e Gram-negativas, incluindo estirpes multi-resistentes. $O$ efeito bactericida das NPs, bem como de nanocompósitos de prata, tem sido intensamente estudado [50].

\section{Conclusões}

O sucesso dos compostos inorgânicos nas práticas médicas tem estimulado novas pesquisas no sentido de desenvolver novos agentes terapêuticos à base de metais. Neste aspecto, a síntese e a avaliação biológica têm conduzido à descoberta de importantes fármacos. Vários complexos de platina, como agentes antitumorais, têm sido obtidos e, considerando as estruturas destes compostos, parece não haver um pré-requisito estrutural. Novos compostos de bismuto vêm sendo obtidos e devido às excelentes actividades citotóxicas demonstradas in vitro e a baixa toxicidade exibida por compostos deste elemento, há a possibilidade dos mesmos serem utilizados como agentes antitumorais, sózinhos ou em associação com outros fármacos. Quanto ao uso de compostos de prata, reforçamos a necessidade de se concentrarem esforços na sua preparação e desenvolvimento, uma vez que podem ser desenvolvidos novos compostos de prata como agentes terapêuticos de uso tópico.

\section{Agradecimentos}

À Universidade Federal de Uberlândia.

\section{REFERÊNCIAS}

[1] C. Orvig, M. J. Abrams, Chem. Rev. 99 (1999) 2201.

[2] A. P. S. Fontes, E. T. César, H. Beraldo, Cadernos Temáticos de Química Nova na Escola: A Química Inorgânica na Terapia do Câncer 6 (2005) 13.

[3] W. Guerra, A. P. S. Fontes, M. V. de Almeida, H. Silva, Quim. Nova 28 (2005) 809.

[4] D. J. Newman, G. M. Cragg, J. Nat. Prod. 70 (2007) 461.

[5] S. M. Cohen, Curr. Op. Chem. Biol. 11 (2007) 115.

[6] C. X. Zhang, S. J. Lippard, Curr. Op.
Chem. Biol. 7 (2003) 481.

[7] S. P. Fricker, Dalton Trans. (2007) 4903.

[8] E. Reisner, V. B. Arion, B. K. Keppler, A. J. L. Pombeiro, Inorg. Chim. Acta 361 (2008) 1569.

[9] J. Kaspárková, O. Nováková, O. Vrána, F. Intini, G. Natile, V. Brabec, Mol. Pharmaco. 70 (2006) 1708.

[10] K. H. Thompson, B. Lichter, C. LeBel, M. C. Scaife, J. H. McNeill, C. Orvig, J. Inorg. Biochem. 103 (2009) 554.

[11] I. Ott, Coord. Chem. Rev. 253 (2009) 1670.

[12] A. Garoufis, S. K. Hadjikakou, N. Hadjiliadis, Coord. Chem. Rev. 253 (2009) 1384.

[13] W. Guerra, E. A. Azevedo, A. R. S. Monteiro, M. Bucciarelli-Rodriguez, E. Chartone-Souza, A. M. A. Nascimento, A. P. S. Fontes, L. L. Moyec, E. C. Pereira-Maia, J. Inorg. Biochem. 99 (2005) 2348.

[14] L., Ronconi, L., P. J. Sadler, Coord. Chem. Rev. 251 (2007) 1633.

[15] P. C. A. Bruijnincx, P. J. Sadler, Curr. Op. Chem. Biol. 12 (2008) 197.

[16] W. Guerra, H. Silva, M. V. de Almeida, A. P S. Fontes, Quím. Nova 30 (2007) 56.

[17] U. Kalinowska-Lis, J. Ochocki, K. Matlawska-Wasowska, Coord. Chem. Rev. 252 (2008) 1328.

[18] F. I. Raynaud, F. E. Boxall, P.M Goddard, M. Valenti, M. Jones, B.A. Murrer, M. Abrams, L. R. Kelland, Clin. Cancer Res. 3 (1997) 2063.

[19] J. Holford, F. Raynaud, B. A. Murrer, K. Grimaldi, J. A. Hartley, M. Abrams, L. R. Kelland, Anticancer Drug Des. 13 (1998) 1.

[20] Y. Chen, Z. Guo, S. Parsons, P. J. Sadler, Chem. Eur. J. 4 (1998) 672.

[21] Y.Chen, J. A. Parkinson, Z. Guo, T. Brown, P. J. Sadler, Angew Chem. Int. Ed. 38 (1999) 2060.

[22] K. A. Gelmon, T. A. Vandenberg, L. Panasci, B. Norris, M. Crump, L. Douglas, W. Walsh, S. J. Matthews, L. K. Seymour, Annals of Oncology 14 (2003) 543.

[23] Y. E. Kwon, K. J. Whang, Y. J. Park, K. H. Kim, Bioorg. Med. Chem. 11 (2003) 1669.

[24] G. Horvath, T. Premkumar, A. Boztas, E. Lee, S. Jon, K. E. Geckeler, Mol. Pharm. 5 (2008) 358.

[25] A. M. Krause-Heuer, N. J. Wheate, M. J. Tilby, D. G. Pearson, C. J. Ottley, J. R. Aldrich-Wright, Inorg. Chem. 47 (2008) 6880. 
[26] N. I. Shtemenko, E. D. Zabitskaya, O. V. Berzenina, D. E. Yegorova, A. V. Shtemenko Chem. Biodiversity 5 (2008) 1660.

[27] D. Moreno, C. T.de llarduya, E. Bandre's, M. Buñuales, M. Azcona, J. García-Foncillas, M. J. Garrido, Eur. J. Pharm. Biopharm. 68 (2008) 503.

[28] I. H. L. Hamelers, A. I. P. M. De Kroon, J. Liposome Res. 17 (2007) 183.

[29] A. P. S. Fontes, Y. Zou, N. Farrel, J. Inorg. Biochem. 55 (1994) 79.

[30] J. D. Roberts, B.Van Houten, Y. Qu, N. Farrel, Nucl. Ac. Res. 17 (1989) 9719.

[31] J. D. Hoeschele, A. J. Kraker, Y. Qu, B. Van Houten, N. Farrell, Molecular Basis of Specificity in Nucleic AcidDrug Interactions, Pullman, B; Jortner, J.; Eds.; Kluwer Academic Publishers, Netherlands, 1990.

[32] N. P. Farrel, Y. Qu, K. Kasparkova, V. Brabec, M. Valsecchi, E. Menta, R. di Domenico, G. da Re, A. Lotto, S. Spi- nelli, Proc. Am. Assoc. Cancer Res. 38 (1997) 2077.

[33] A. L. Harris, J. J. Ryan, N. Farrell, Mol. Pharmacol. 69 (2006) 666.

[34] N. Farrel, Y. Qu, M. P. Hacker, J. Med. Chem. 33 (1990) 2179.

[35] M. S. P Ladeira, D. M. F. Salvadori, M. A. M. Rodrigues, J. Bras. Patol. Med. Lab. 39 (2003) 335.

[36] G. G. Briand, N. Burford, Chem. Rev. 99 (1999) 2601.

[37] K. Nomiya, K. Sekino, M. Ishikawa, A. Honfa, M. Yokoyama, N. C. Kasuga, S. Nakano, K. Onodera, J. Inorg. Biochem. 98 (2004) 601.

[38] S. Ahmad, A. A. Isab, S. Ali, A. R. AlArfaj, Polyhedron 25 (2006) 1633.

[39] I. Turel, L. Golie, P. Bukovec, M. Gubina, J. Inorg. Biochem. 71 (1998) 53.

[40] A. R. Shaikh, R. Giridhar, M. R. Yadav, Int. J. Pharm. 332 (2007) 24.

[41] E. R. T. Tiekink, Crit. Rev. Oncol. Hematol. 42 (2002) 225.
[42] K. luchi, Y. Hatano, T. Yagura, Biochem. Pharmacol. 76 (2008) 974.

[43] X. Y. Wang, X. M. Zhang, J. Lin, J. W. Chen, Q. Xu, Z. J. Guo, J. Chem. Soc. Dalton Trans. (2003) 2379.

[44] R. Huang, A. Wallqvist, D. G. Covell, Biochem. Pharmacol. 69 (2005) 1009.

[45] J. G. Jurcic, Crit. Rev. Oncol. Hematol. 38 (2001) 37.

[46] I. Chopra, J. Antimicrob. Chemother. 59 (2007) 587.

[47] L. L. Marques, G. M. Oliveira, E. S. Lang, M. M. A. Campos, L. R. S. Gris, Inorg. Chem. Commun. 10 (2007) 1083.

[48] M. S. Refat, Spectrochim Acta Part A 68 (2007) 1393.

[49] M. Raí, A. Yadav, A. Gade, Biotechnology Advances 27 (2009) 76.

[50] B. Ruan, Y. Tian, H. Zhou, J. Wu, Z. Liu, C. Zhu, J. Yang, H. Zhu, J. Org. Chem. 694 (2009) 2883.

\section{Actualidade Científica}

\section{ZEÓLITOS COM POROS EQUILIBRADOS}

De acordo com investigadores do Instituto de Investigação Química da Catalunha, o tratamento de zeólitos com uma solução alcalina e catiões orgânicos relativamente grandes, promove a formação de uma estrutura cristalina porosa caracterizada por uma distribuição de tamanhos de poros idealmente adequados para aplicações catalíticas (Adv. Funct. Mater. 19 (2009) 1).

Os zeólitos são aluminossilicatos cristalinos e porosos, largamente usados como catalisadores na refinação do petróleo. Durante anos, os investigadores procuraram formas de preparar zeólitos com poros largos (mesoporos) para melhor acomodar moléculas grandes e permitir o fluxo de reagentes e de produtos para o interior e o exterior dos cristais.

Nesse sentido, foram desenvolvidos vários métodos, no entanto, muitos formam poros largos por eliminação de poros mais pequenos (microporos), onde frequentemente se localizam os centros activos catalíticos do material, afectando por isso a sua performance catalítica.

Recentemente, uma equipa de investigadores liderada por Javier Pérez-Ramírez, anunciou que o tratamento do zeólito ZSM-5 com hidróxido de sódio na presença de iões tetrapropil- ou tetrabutilamónio $\left(\mathrm{TPA}^{+}\right.$e $\mathrm{TBA}^{+}$, respectivamente) aumenta o tamanho dos microporos maiores sem sacrificar os microporos mais pequenos.

A equipa explica que os iões hidróxido lixiviam a sílica do interior dos microporos, aumentando por isso o seu tamanho. Mas o crescimento dos poros, que até esta descoberta era difícil de controlar, é moderado pelos catiões $\mathrm{TPA}^{+}$e $\mathrm{TBA}^{+}$, que adsorvem à entrada dos poros, impedindo a lixiviação do hidróxido.

Para quantificar os benefícios catalíticos do novo método, o grupo da Catalunha comparou zeólitos preparados de várias formas por um parâmetro que desenvolveram, o factor hierár- quico (HF), que representa o produto entre o volume de microporos e a área superficial mesoporosa.

Em testes reaccionais efectuados, a equipa concluiu que os catalisadores mais produtivos eram os zeólitos com os valores de HF mais elevados. (adaptado de Chemical \& Engineering News - http://pubs.acs.org/cen/ news/87/i46/8746news8.html, acedido em 19/11/2009)

Helder Gomes 